\title{
Modeling of Material Removal Morphology And Prediction of Surface Roughness Based On WEDM Successive Discharges
}

Zhaolong Li ( $\sim$ lizhaolong@hrbust.edu.cn )

Harbin University of Science and Technology

Yingtao Liu

Harbin University of Science and Technology

Bingren Cao

Harbin University of Science and Technology

Wangwang Li

Harbin University of Science and Technology

\section{Research Article}

Keywords: WEDM, Successive discharges, FEM, Modeling, Surface roughness prediction.

Posted Date: November 29th, 2021

DOI: https://doi.org/10.21203/rs.3.rs-1113386/v1

License: (a) (i) This work is licensed under a Creative Commons Attribution 4.0 International License. Read Full License

Version of Record: A version of this preprint was published at The International Journal of Advanced Manufacturing Technology on February 17th, 2022. See the published version at https://doi.org/10.1007/s00170-022-08870-5. 


\title{
Modeling of material removal morphology and prediction of surface roughness based on WEDM successive discharges
}

\author{
Zhaolong $\mathrm{Li}^{1,2}$, Yingtao $\mathrm{Liu}^{2}$, Bingren $\mathrm{Cao}^{2}$, Wangwang $\mathrm{Li}^{2}$ \\ 1 Key Laboratory of Advanced Manufacturing Intelligent Technology of Ministry of Education, Harbin \\ University of Science and Technology, Harbin, Heilongjiang Province 150080, China \\ 2 School of Mechanical and Power Engineering, Harbin University of Science and Technology, Harbin, \\ Heilongjiang Province 150080, China
}

Corresponding Author: lizhaolong@hrbust.edu.cn

\begin{abstract}
In this paper, a successive discharges material removal model is proposed to predict the surface roughness of the EN-GJL-250 (Grey Cast Iron) under different discharge parameters of Wire-cut electrical discharge machining (WEDM). Firstly, the finite element method (FEM) was used to analogue simulation the distribution of material surface temperature and flow field in the WEDM single pulse discharge machining process. According to the principle of energy conservation, the surface morphology and characteristic parameter size of a single pulse crater under different discharge parameters are simulated. Secondly, the discharge position is determined by the minimum gap width between the initial surface of the workpiece and the electrode wire during the establishment of the material removal model. Simulation results show: The magnitude of the single pulse discharge energy has an important influence on the morphology and characteristic size of the crater. Due to the different discharge energy, the increasing trend of crater radius, depth and flanging height is not synchronized, and the increase of crater radius is more obvious and rapid. Meanwhile, the final morphology of the WEDM machined surface is determined by the morphology size of a single pulse crater and the location distribution of discharge points. Finally, the same discharge parameters are used to verify the surface quality of machined materials. Experimental results show: The surface morphology of the WEDM workpiece is in good agreement with the material removal model, and the average relative error of surface roughness between the simulation results and the experimental results is $8.26 \%$.
\end{abstract}

Keywords: WEDM; Successive discharges; FEM; Modeling; Surface roughness prediction.

\section{Introduction}

Cast iron material has the characteristics of high strength, wear resistance and heat resistance. In all cast iron materials EN-GJL-250(Grey Cast Iron) because of its corrosion resistance, shock absorption, and excellent casting performance and other characteristics, often used in the manufacture of some machine tool bed, column and gear parts. WEDM uses the instantaneous local high temperature generated by the pulse spark discharge between the workpiece and the electrode wire to melt and vaporize the workpiece material, to achieve the purpose of removing the material. Compared with the traditional machining, WEDM makes the electrode wire keep a certain distance from the workpiece, and can achieve high precision 
machining [1-5]. When machining cast iron materials by WEDM, not only the surface deformation of the workpiece is small, there is almost no environmental pollution in the whole machining process. These characteristics make WEDM very suitable for machining EN-GJL250 materials [6-9]. However, due to the randomness and disorder in the WEDM process, the surface roughness of processed materials often cannot reach the predetermined standard, and the processing of materials cannot be directly observed by instruments and equipment in the process of machining, so the surface roughness of processed materials cannot be accurately controlled. In the WEDM process, discharge parameters play an important role in surface roughness, many researchers control surface roughness by optimizing discharge parameters [10-13]. Taguchi method is widely used to optimize the WEDM process, and the optimal parameters can be obtained through experiments and statistical analysis. Kunieda et al. [15] conducted basic research on WEDM from the aspects of mechanical and electrical principles, and provided basic insights on WEDM processing. Chiang and Chang et al. [16] studied the effects of WDEM process parameters such as the cutting radius of workpiece, water flow and the wire feed rate on the surface roughness of alumina particle reinforced materials, and optimized the process parameters. Chaudhari, et al. [17] used the heat transfer search algorithm to conduct multi-response optimization of process parameters for WEDM machining super-elastic Ni-Ti-N shape memory alloy. At present, based on the principle of WEDM, the establishment of a material removal model based on successive discharges is always considered to be a challenging job. Furthermore, very few works have been conducted on the modeling of surface roughness due to the WEDM process.

To model WEDM processes, various comprehensive mathematic models and simulation methods have been established. Tao and $\mathrm{Ni}$ et al. [18] established a model to simulate the discharge crater formation by considering the effect of bubble pressures to get a well-matched crater geometry in an EDM process. Yue and Yang et al. [19] used the molecular dynamics method to simulate the formation of craters in EDM under the condition of considering the pressure of the medium between the electrodes. It is found that the higher the medium pressure, the higher the material removal rate. The establishment of a single pulse discharge crater model in WEDM is of great significance to study the formation of final surface morphology[2022]. However, the formation of a single crater is not enough to explain the actual WEDM process, in which the workpiece surface is generated by the superposition of craters formed by multiple discharges [23-25]. Izquierdo et al. [26] have performed a numerical study based on the finite difference method. By using inverse identification, they have estimated the material removal rate and surface roughness within a $6 \%$ error margin. Jithin et al. [27] established a surface topography prediction model under EDM continuous discharge based on FEM, and $\mathrm{Ra}$ simulation results were consistent with experimental results. Wang et al. [28] established a model based on $3 \mathrm{D}$ continuous discharge to study the relationship between workpiece surface topography and electrode wire vibration. Results: In the SV-MF composite assisted WEDM process, the surface roughness of the workpiece decreases by more than $10 \%$, and the 
uniformity of the discharge position distribution is improved. The simulation results are verified by experiments and the simulation error of surface roughness is less than $5 \%$.

In this paper, a 3D material removal model is proposed to simulate the successive discharges machining process of WEDM. The relationship between single pulse crater morphology, discharge position and dynamic surface morphology is established. Meanwhile, the effects of the molten vaporization of the processed material and the residual heat generated by a single spark are considered for simulation. The effects of different discharge energies on the surface morphology and size of the impact crater are studied, and a single pulse discharge crater model is established. The discharge position is determined by the minimum gap width, rather than assuming a random distribution of discharge position, which is more consistent with the actual WEDM machining process. Several groups of profile lines were extracted from the $3 \mathrm{~d}$ material removal model, and the characteristic parameters were determined by means of averaging, to predict the surface roughness. In this study, pulse width and peak current were used as input parameters, and surface roughness was used as output parameters. Experiments verified the accuracy of the continuous discharge material removal model in predicting surface roughness.

\section{Study on thermal-flow coupling} simulation model of WEDM single pulse discharge

In this study, COMSOL Multiphysics 5.3 (COMSOL Inc., Burlington, MA, MSA), a commercial finite element software package, was used to model the temperature and flow field during the WEDM machining process.
WEDM uses ultra-high thermal erosion to rapidly increase the surface temperature of the workpiece, and make it melt or even vaporize and evaporate. Therefore, there is a mutual transformation of the solid phase, liquid phase and gas phase of metal materials on the surface of the workpiece during single pulse discharge, as shown in Fig. 1. Since molten/vaporized materials exhibit fluid heat transfer and flow characteristics, which not only involve changes in temperature distribution, but also include changes in flow velocity and pressure. Therefore, the simulation method of coupled analysis of thermal field and flow field can more accurately simulate the morphology of discharge crater.

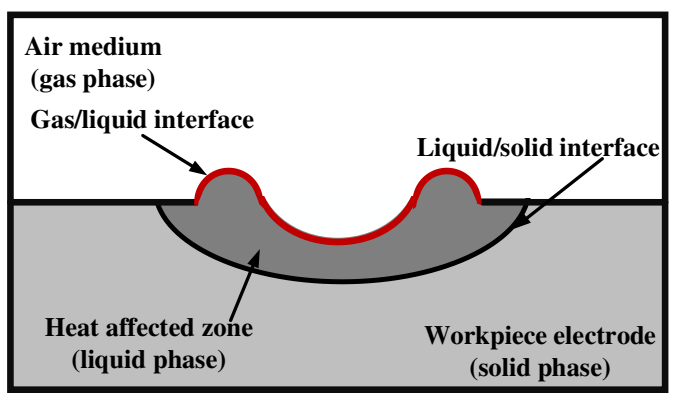

Fig. 1 Schematic diagram of regional phase transformation in discharge crater.

In WEDM machining, an instantaneous high temperature heat source is formed on the surface of the workpiece after the breakdown of the interelectrode medium. At this time, the discharge channel is in an extremely high temperature state, and the heat energy in the discharge channel is continuously transmitted to the surface of the workpiece. Finally, the discharge crater is formed. To reduce the calculation amount of simulation, the following assumptions are made on the model :

1. The workpiece and electrode wire are uniform and isotropic ;

2. Assuming that a pulse discharge produces only one discharge pit, the discharge energy is 
mainly transferred by heat conduction and heat convection, and the thermal radiation is ignored.

3. The discharge channel is cylindrical, and the radius of the discharge channel is a function of the pulse width and the discharge current. The heat flux on the electrode surface is Gaussian distribution, and the action position is axisymmetric.

4. Solid/molten/vaporized materials are regarded as continuous medium and Newtonian viscous fluid, air and metal vapor are regarded as the same gas phase, and the flow of molten/vaporized materials is regarded as incompressible laminar flow.

5. The thermophysical parameters and flow characteristics of the material are functions of temperature.

\subsection{Heat source model}

In the process of WEDM machining, heat conduction is the main way of heat transfer in the workpiece, electrode and plasma channel. At present, it is generally believed that the surface heat source with Gaussian distribution is closer to the thermal erosion effect produced in actual machining, and the heat source radius of the discharge center is roughly the same as the radius of the discharge channel. Therefore, the surface heat source with Gaussian distribution law is loaded on the surface of the workpiece as the equivalent heat source of the discharge channel for thermal energy input. The heat flux density in the discharge channel can be calculated according to Eq. 1. Based on the comprehensive consideration of previous research results and positive polarity processing characteristics of anode workpiece materials, the energy distribution ratio of the anode is set as $30 \%$ of the total discharge energy in this paper [29, 30]. $q(r, t)=\frac{4.55 \eta u(t) i(t)}{\pi R^{2}(t)} \exp \left(-4.5 \frac{r^{2}}{R^{2}(t)}\right)$

Where $q(r, t)$ is the heat flow density $\left(\mathrm{W} / \mathrm{m}^{2}\right)$ at radius $r$ at time $t ; r$ is the radial position (m) away from the heat source center; $R(t)$ is timevarying radius (m) of discharge channel; $\eta$ is the heat distribution coefficient; $u(t)$ is the discharge voltage $(\mathrm{V}) ; i(t)$ is the discharge current $(\mathrm{A})$.

\subsection{Discharge channel radius}

Discharge channel radius is an important factor in temperature field simulation, but it is difficult to measure its specific value through experiment because of the short discharge duration and small discharge gap[31]. To describe the expansion and variation of discharge channel radius, the discharge channel radius is calculated according to Eq. 2 .

$R=2.04 i^{0.43} t_{\mathrm{on}}^{0.44}$

Where $R$ is the discharge channel radius (m); $i$ is the discharge current $(\mathrm{A}) ; t_{o n}$ is pulse width (s);

\subsection{Physical model and meshing}

In order to obtain more accurate simulation results, it is necessary to apply the same model boundary conditions as the actual machining process to the model, so that the numerical calculation results are in good agreement with the actual machining situation. The model boundary conditions are shown in Fig. 2a Boundary conditions of thermal interaction between workpiece and surrounding medium are shown in Eq. 3.

$k \frac{\partial T}{\partial z}=\left\{\begin{array}{cl}q(r, t) & r \leq R(t) \\ h_{c}\left(T-T_{o}\right) & r>R(t) \\ 0 & \text { else }\end{array}\right.$

Where $T$ is the workpiece temperature $(\mathrm{K}) ; h_{c}$ is the convective heat transfer coefficient $\left(\mathrm{W} / \mathrm{m}^{2} / \mathrm{K}\right) ; T_{o}$ is the ambient temperature $293.15 \mathrm{~K}$.

Meanwhile, to improve the efficiency of the simulation, a two-dimensional axisymmetric 
model is used for modeling and analysis. The simulation research area mainly includes two domains: interelectrode medium domain and workpiece electrode domain, and their calculated size ranges are $300 \mu \mathrm{m} \times 100 \mu \mathrm{m}$ and $300 \mu \mathrm{m} \times 200 \mu \mathrm{m}$, respectively. The axis of symmetry of the model is AOE, which represents the central axis of the discharge channel; Point $\mathrm{O}$ is the loading center of Gaussian heat source, OF area is the heat source

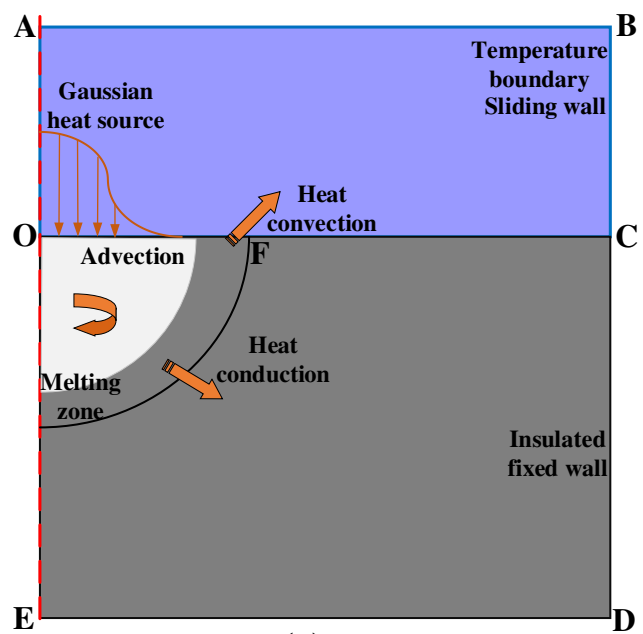

(a)

Fig. 2 (a) Model boundary condition setting; (b) Grid division result.

\subsection{Governing equations of the model}

The influence factors of the thermal-flow coupling simulation model are as follows: the thermal phase transformation caused by heating of materials, the heat transfer and flow of molten fluid materials, and the mass loss caused by the instantaneous evaporation of materials. Therefore, the control equation includes the heat transfer equation with convection term, NavierStokes equation and continuity equation.

According to the energy conservation theorem, the temperature distribution on the workpiece surface is calculated by Eq. 4 .

$\rho C_{p} \frac{\partial T}{\partial t}+\rho C_{p} u \cdot \nabla T+\nabla \cdot(-k \nabla T)=Q+Q_{v a p}$

Where $\rho$ is the density of material $\left(\mathrm{kg} / \mathrm{m}^{3}\right), C_{p}$ is

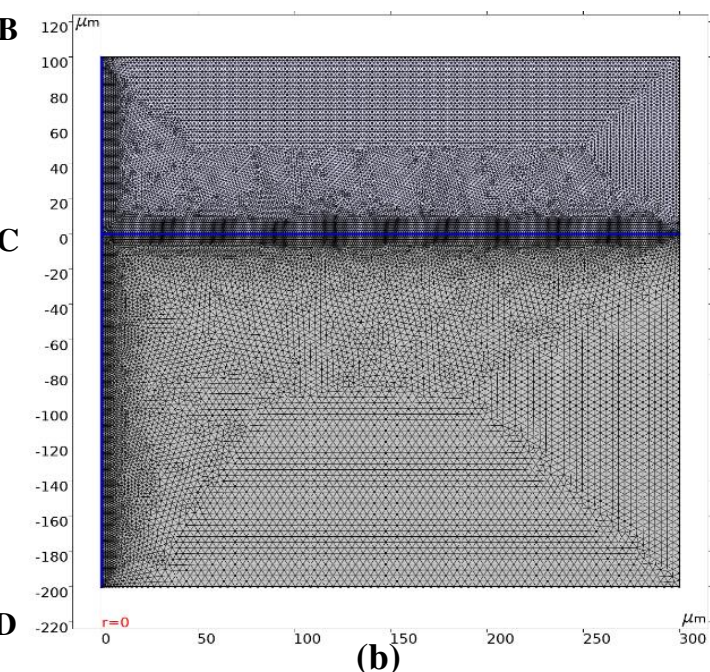

(b) range, apply heat convection to the FC area. Since the size of the selected calculation model is much larger than that of the discharge crater, the workpiece can be considered as a semiinfinite region. To improve the accuracy of simulation calculation, the method of nonuniform meshing combined with adaptive meshing was adopted for meshing, and the finite element model obtained was shown in Fig. 2 b.

the specific heat capacity of materials $(\mathrm{J} / \mathrm{kg} / \mathrm{K}), t$ is the computation time (s), $u$ is the velocity of flow of fluid material $(\mathrm{m} / \mathrm{s}), k$ is the thermal conductivity of materials $(\mathrm{W} / \mathrm{m} / \mathrm{K}), Q$ is the Gaussian heat source term $\left(\mathrm{W} / \mathrm{m}^{2}\right), Q_{v a p}$ is the phase change energy loss source term $\left(\mathrm{W} / \mathrm{m}^{2}\right)$.

According to the momentum conservation theorem, the flow velocity and pressure of molten / vaporized materials are calculated by Eq. 5.

$$
\begin{aligned}
\rho \frac{\partial u}{\partial t}+\rho(u \cdot \nabla) u & =\nabla \cdot\left[-P I+u\left(\nabla u+(\nabla u)^{T}\right)\right] \\
& +F+\rho g
\end{aligned}
$$

Where $I$ is the unit matrix, $u$ is the dynamic viscosity of fluid materials $(\mathrm{Pa} \cdot s), P$ is the fluid material pressure $(\mathrm{Pa}), F$ is the volumetric force 
source term, $F=F_{s t}+F_{\tau}+F_{P}+F_{v}+F_{D}$.

According to the mass conservation theorem, the mass loss caused by material evaporation is calculated by Eq. 6 .

$\nabla \cdot u=m_{s}$

Where $m_{s}$ is the evaporation mass loss source term $\left(\mathrm{W} / \mathrm{m}^{2}\right)$.

\section{Simulation results and analysis}

\subsection{Temperature field distribution and flow} field change rule in the discharge area

In a single pulse discharge cycle, the temperature field distribution of the crater area generated on the workpiece surface is shown in Fig. 3. At $1 \mu \mathrm{s}$, the temperature of the high temperature overheating zone on the surface of cast iron is very high, and the heat is concentrated, but the heat-affected zone is small. With the increasing discharge time and the distance between the discharge area and the heat source center, the temperature increases rapidly and evenly, and the heat absorbed by the surface of the workpiece is continuously transferred to the internal, resulting in the gradual increase of the internal temperature of the workpiece. In the range of $5 \mu$ s to $20 \mu \mathrm{s}$, the surface temperature of the workpiece decreases, because the expansion of the discharge channel radius leads to the decrease of energy density in the discharge region, and the phase transformation and vaporization of the material also cause heat loss. At $100 \mu$ s, the single spark discharge ended, but under the influence of the discharge residual heat, the surface of the material gradually appeared "earlobe shape" temperature expansion trend, until $160 \mu$ s to reach stability. This is because the cooling effect of the working fluid causes the temperature in the region outside the discharge channel radius to decrease rapidly. At the same time, it is found that the transfer rate of heat along the radius direction is always greater than that in the depth direction in different discharge periods.

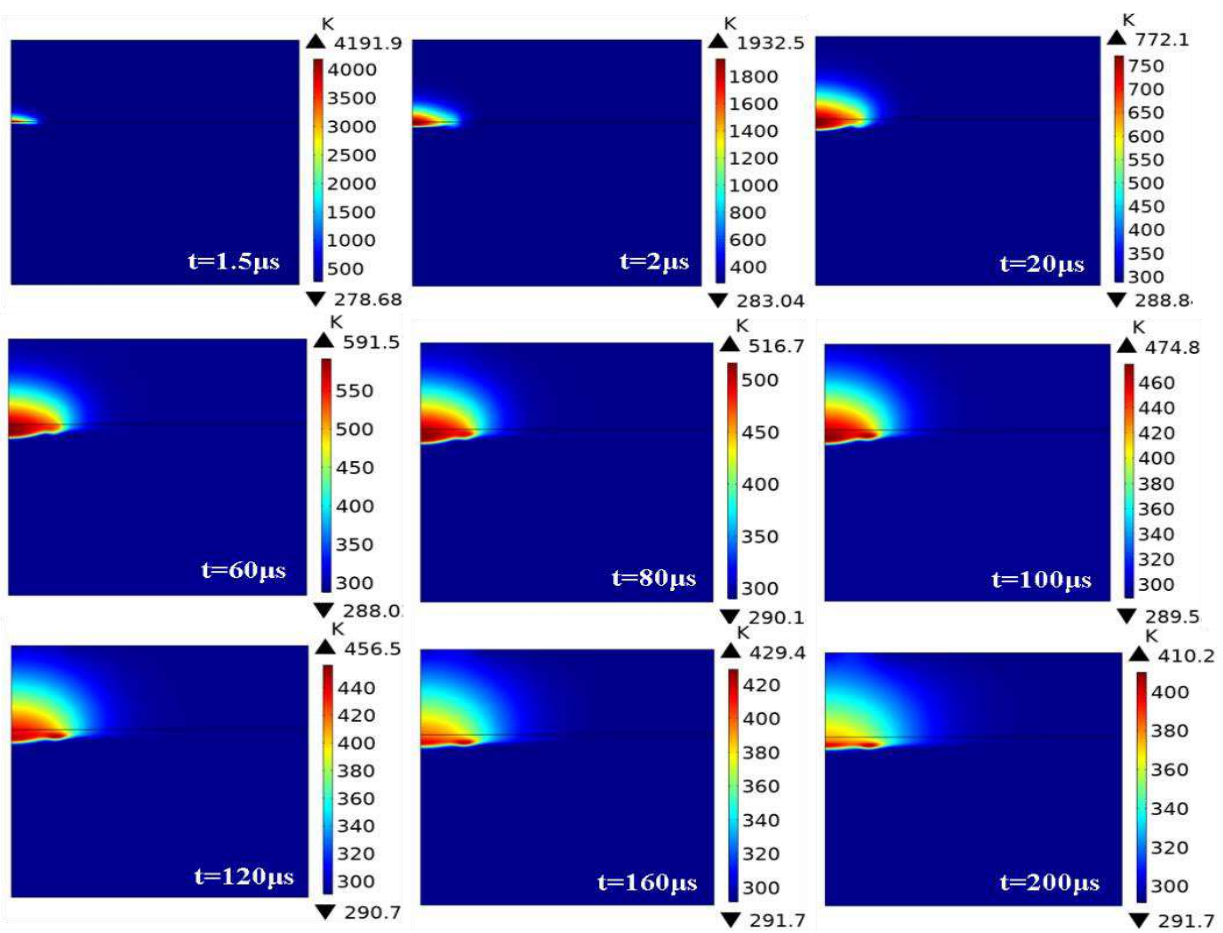

Fig. 3 Temperature distribution in the single pulse discharge crater area at different times. 
Fig. 4 and Fig. 5 show the variation trend of temperature and flow velocity in the radius and depth direction of the discharge area in the single pulse discharge cycle. It can be seen from Fig. 4 that in the radial region, the closer to the discharge center, the higher the temperature, and the farther away from the discharge center, the lower the temperature, which is consistent with the Gaussian distribution of heat source in actual processing. The temperature distribution in the depth direction has the same trend, and the temperature gradient in the depth direction is larger than that in the radius direction. It can be seen from Fig. 5 that the flow velocity distribution in the radius direction is different from the temperature distribution. The velocity

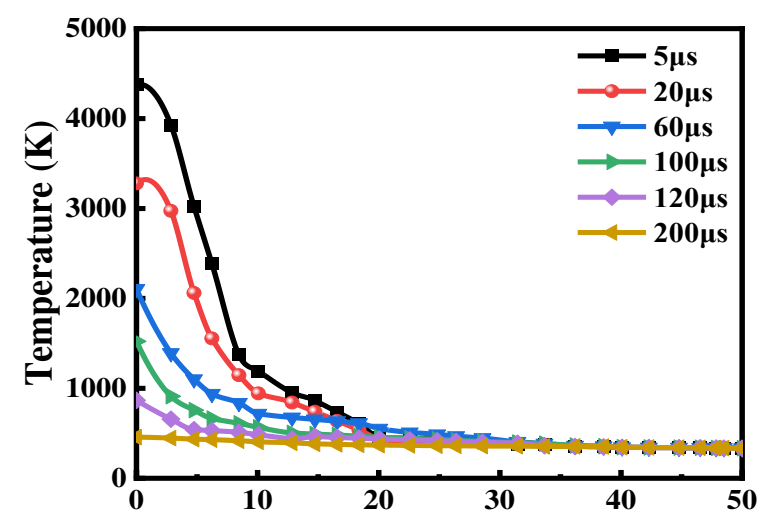

(a) Radial position $(\mu \mathrm{m})$ in the radius direction increases first and then decreases with the increase of the horizontal distance of discharge center. As calculated by Eq. 4, the maximum velocity point always appears near the boundary of the final shape of the pit. Analysis of the reasons: under the combined effect of material steam flow and discharge heat, the superheated molten material at the bottom of the crater flows to the edge of the crater, and the crater sinks inward. The overall variation of velocity in depth direction is consistent with that in radial direction, but its variation is more complicated. This is because in the discharge process, there is not only material melting and material gasification, material evaporation also affects the flow of hot melt material.

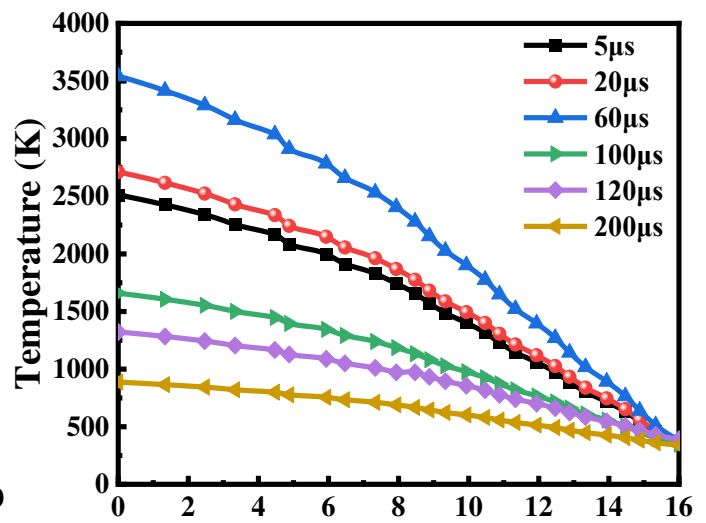

(b) Depth-direction position $(\mu \mathrm{m})$

Fig.4 Temperature distribution trend of single pulse discharge crater; (a) in the radius direction (b) in the depth direction.

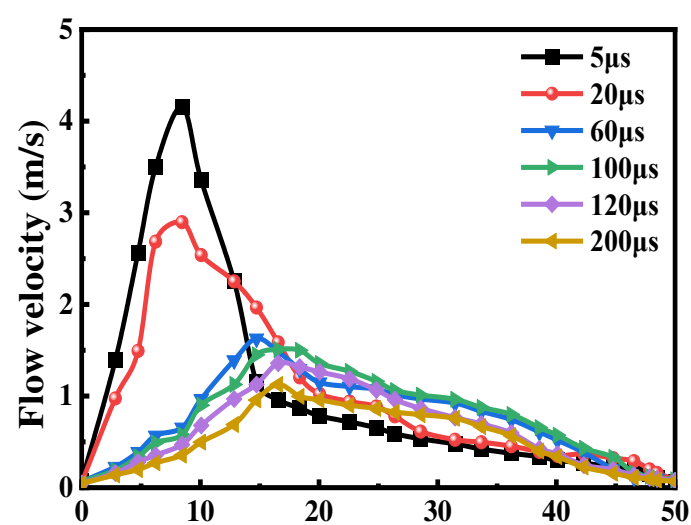

(a) Radial position $(\mu \mathrm{m})$

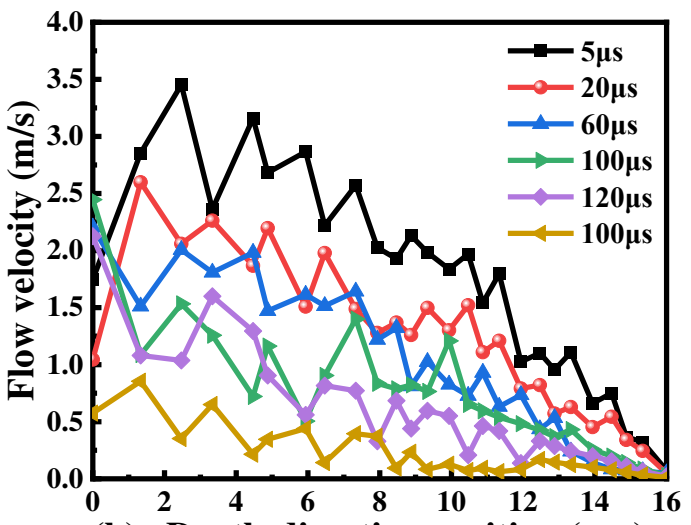

(b) Depth-direction position $(\mu \mathrm{m})$

Fig. 5 Velocity variation trend of single pulse discharge crater; (a) in the radius direction (b) in the depth direction. 


\subsection{Formation process of single pulse}

\section{discharge crater}

In the single pulse discharge cycle, the change process of single crater topography on EN-GJL-250 material surface is shown in Fig. 6. As can be seen from Fig. 6, within $1.5 \mu$ s of initial discharge, the surface of the material has a tendency to form meteorite crater morphology, and the diameter and depth of meteorite crater do not increase significantly. With the increase of discharge energy, in the range of $5 \mu$ s to $60 \mu \mathrm{s}$, the crater diameter expands obviously, but the depth increases slowly. From $60 \mu$ s to $100 \mu$ s, the crater diameter slowly expands and the depth begins to increase, and the increase rate is more obvious than that of the first $60 \mu \mathrm{s}$. At $100 \mu \mathrm{s}$, the

single pulse spark discharge ended, but the crater size still showed an increasing trend, indicating that after the discharge, due to the existence of high temperature heat on the surface of the material, the material erosion did not stop immediately, but continued for a period of time, until $150 \mu$ s, the diameter and depth of the crater began to remain stable. In the range of $10 \mu$ s to $80 \mu$ s, a relatively obvious bump appeared on the crater edge, and then the bump gradually became sharp. At the end of the discharge, the flanging bulge gradually becomes sharp and then smooth. In the range of $180 \mu$ s to $200 \mu \mathrm{s}$, the height of the flanged bump decreases due to the re-flow of uneroded molten material into the pit.
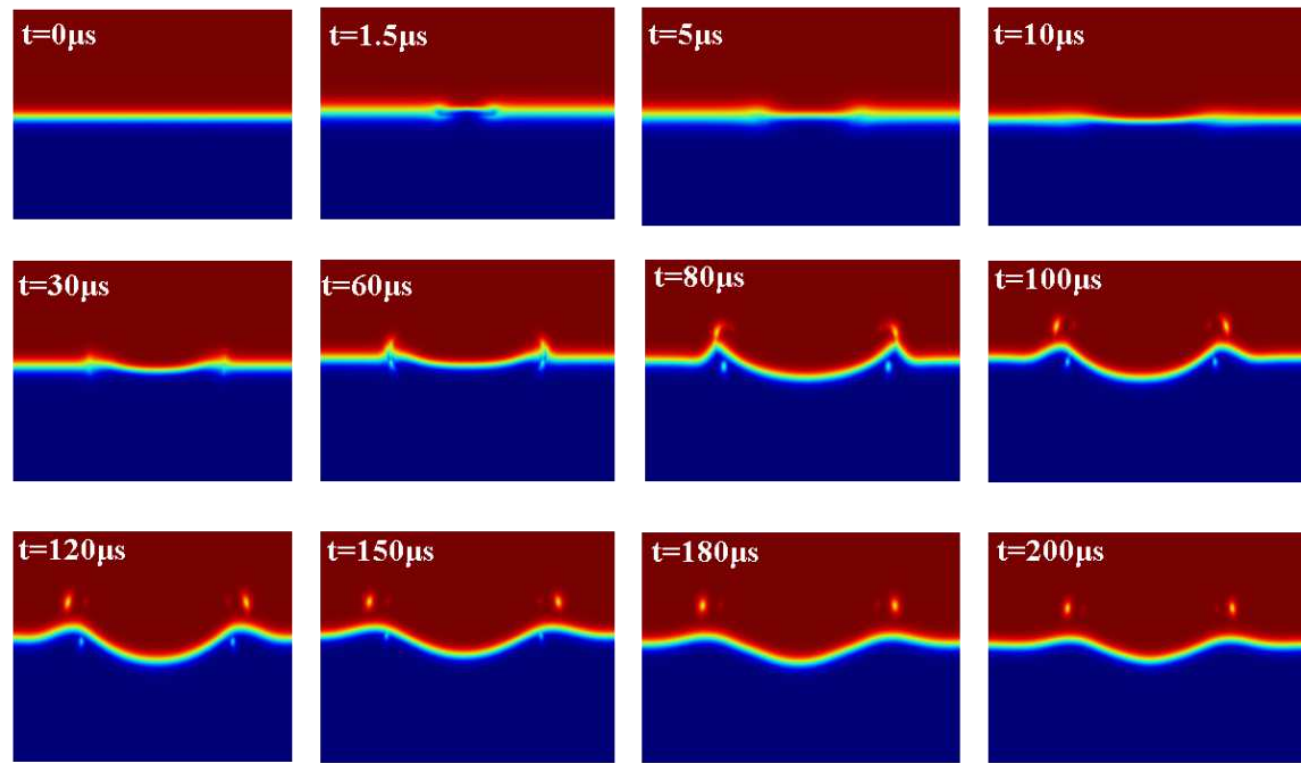

$\mathbf{\nabla}$
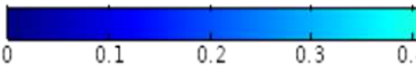

0.5

Fig. 6 Formation process of single pulse discharge crater.

To sum up, in the process of single pulse crater formation, the molten material will first form a smooth depression in the center and then be pushed to both sides and continuously eroded, at the same time, the edge of the crater flanging bulge. Under the influence of residual heat generated by a single spark, the diameter, depth and flanging height of the crater firstly increased and then decreased for a period of time.

\subsection{Impact of discharge parameters on crater feature size}

In the process of electrothermic-flow 
coupling simulation of single pulse discharge, to more intuitively analyze the change rule of morphology and characteristic size in the formation process of a meteorite crater, numerical simulation was carried out on the size of a meteorite crater under different discharge parameters, and the single pulse discharge crater model was established, as shown in Fig. 7.

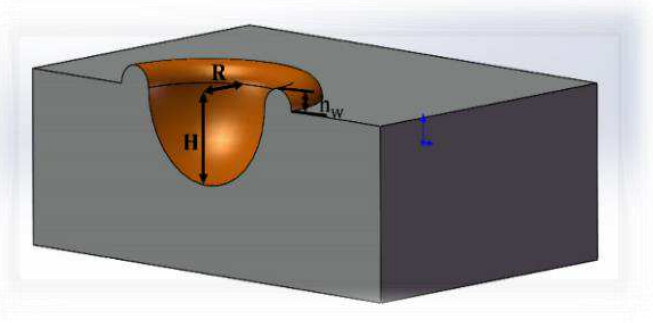

Fig. 7. Single pulse discharge crater model.

According to the energy conservation theorem, the variation of crater radius $\mathrm{R}$, depth $H$ and flanging height $h_{w}$ under different discharge currents and pulse widths is shown in Fig. 8. From Fig. 8a, it can be found that the depth of the single pulse discharge crater increases rapidly with the increase of the peak current, and then slowly decreases; As the pulse width increases, it shows a sharp rise and then a sharp drop. The crater depth reaches a peak value of $13.40 \mu \mathrm{m}$ when the peak current is $14 \mathrm{~A}$ and the pulse width is $32 \mu \mathrm{s}$. It can be found from Fig. $8 \mathrm{~b}$ that the radius of a single pulse crater increases with the increase of the peak current and pulse width, and the influence of the peak current on the crater radius is much greater than the pulse width. From Fig. 8c, it can be found that the flanging height of the single pulse discharge crater is most affected by the pulse width. With the increase of pulse width and peak current, the flanging height first increases and then decreases. When the peak current is $8 \mathrm{~A}$ and the pulse width is $52 \mu \mathrm{s}$, it reaches the peak value of $13.4 \mu \mathrm{m}$.
In the summary: In the process of crater formation, with the increase of peak current and pulse width, the increasing trend of crater radius, depth and flanging height is not synchronous, and the increase of crater radius is more obvious and rapid. The crater radius first reaches the maximum value and remains stable, then the crater depth reaches the stable value, and the flanging height reaches the stable value at the slowest rate.

\section{(a) Crater depth $(\mu \mathrm{m})$}

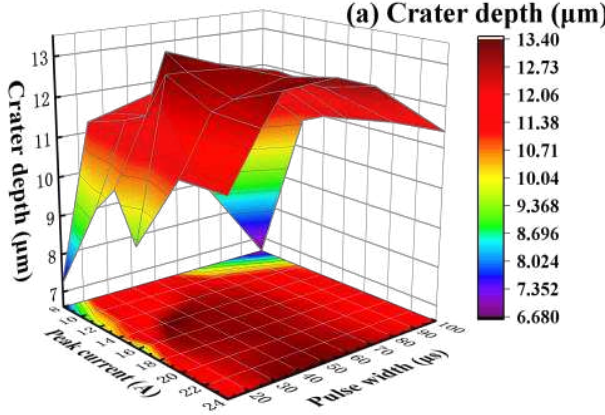

(b) Crater diameter $(\mu \mathrm{m})$

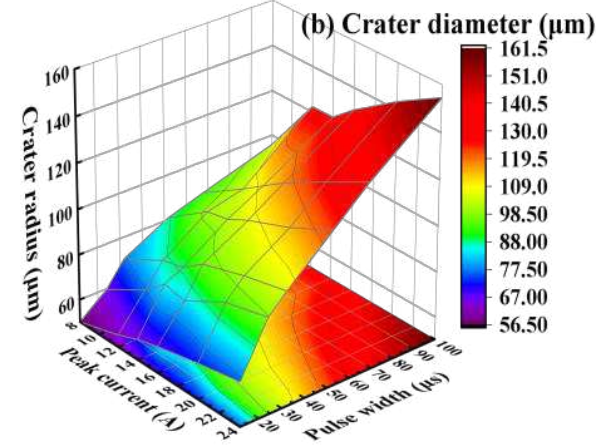

(c) Height of crater flanging $(\mu \mathrm{m})$

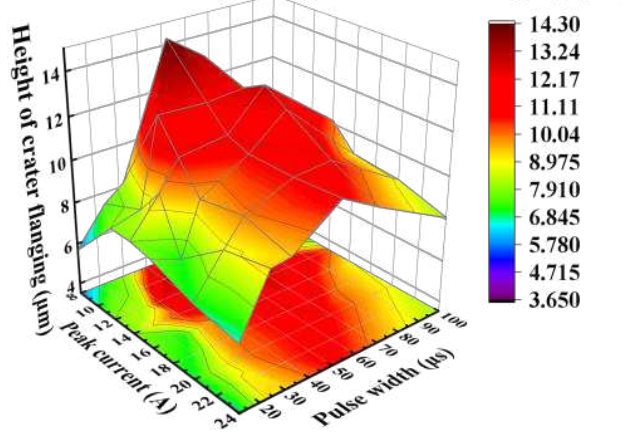

Fig. 8 (a) Fitting relationship of crater depth with discharge current and pulse width ; (b) Fitting relationship of crater diameter with discharge current and pulse width; (c) Fitting of crater flanging height with discharge current and pulse width. 


\section{Establishment of continuous discharge material removal model in WEDM}

The above simulation shows the influence of discharge energy on the morphology and size of the crater during the single pulse discharge process. In the actual machining process, the material is removed by continuous discharge machining. This means that according to the discharge position superimposed meteorite crater can simulate the new surface appearance. However, the distribution of discharge position is random and irregular, which is affected by many factors. Therefore, this paper presents a novel model to predict surface topography. The following assumptions are made to simplify the model:

1. The workpiece material and dielectric fluid are homogeneous and isotropous.

2. Ignoring the Influence of Electrode Wire Vibration on Discharge Position.

3. During the discharge process, the discharge radius in the plasma channel remains constant.

4. The morphology and size of the single crater generated by each pulse discharge are consistent.

To simplify the model, deionized water on the surface is regarded as the optimal flushing condition. Under this circumstance, it could be accepted that the most influential factor on discharge location is the gap width because a smaller gap width leads to a greater electric field intensity. Assuming that the expression of the initial workpiece surface is $\varphi(x, y, z)$, the expression of the position of the electrode wire is $\delta(x, y, t)$. The discharge location is determined by searching the minimum gap width, which is given as:

$\left[\begin{array}{c}(a, b, c) \\ \varepsilon\end{array}\right]=\min (|\varphi(x, y, z)-\delta(x, y, t)|)$

Where $|\varphi(x, y, z)-\delta(x, y, t)|$ is the distance between the surface topography and the wire electrode, $(a, b, c)$ is the relative position of discharge location on the workpiece, $\varepsilon$ is the position of discharge location on the wire electrode. After the first discharge position is determined, the discharge position on the initial surface of the workpiece and the electrode wire can be calculated for the next discharge. Therefore, the surface topography of the workpiece after a series of continuous discharges can be expressed as:

$\omega(x, y, z)=\varphi(x, y, z)-\sum_{i=1}^{n} f\left(\left(x-a_{i}\right),(y-\right.$ $\left.\left.b_{i}\right),\left(z-c_{i}\right)\right)$

Where $f(x, y, z)$ is Shabgard and Gholipoor et al. [32] proposed a calculation formula for the single pulse discharge crater model. The continuous discharge process is shown in Fig. 9.

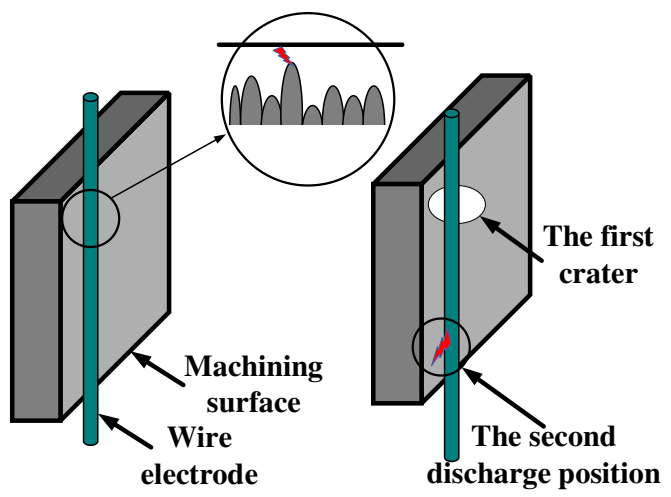

Fig. 9 Successive discharge process.

The material removal model established in this study does not assume the number and random distribution of discharge positions, and the discharge positions are determined by calculating the minimum gap width between the initial surface of the workpiece and the electrode wire. Single pulse craters are then superposed in a sequence. Thus, the simulated workpiece surface topography is more consistent with the actual WEDM machining process.

\subsection{Calculation of surface roughness of material removal model}

The surface topography of the workpiece 
after WEDM is the result of a series of overlapping discharge craters. The roughness of the workpiece surface is determined by crater size and location [33-34]. Since the distribution of WEDM discharge position is random and irregular, the prediction of surface roughness is not accurate either by continuous and nonoverlapping crater distribution [35] or by continuous overlap between adjacent craters with a distance of $\mathrm{D} / 8$ ( $\mathrm{D}$ is the diameter of discharge pits) [36]. Salonitis et al. [37] uniformly arranged discharge craters on the plane, removed the intersection part of craters, and calculated the surface roughness with the residual part. The simplified model is shown in Fig. 10. Zhang et al. [38] observed the experimental samples by electron microscope, and measured the value of $a$, which is $1 / 3$ of $s$.

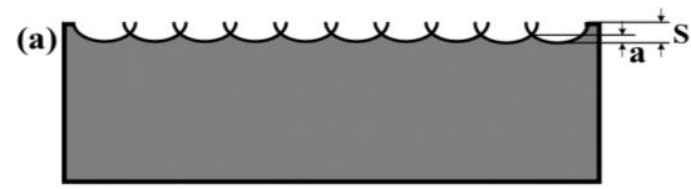

(b)

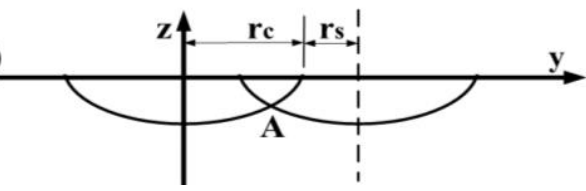

Fig. 10 Simplified model of surface roughness calculation.

The calculation formula of surface roughness is as follows:

$R_{a}=\frac{1}{4}\left[\frac{r_{c}+r_{s}}{r_{c}}\right]^{2} \cdot S$

Where $s$ is the distance between the initial plane and the crater bottom, corresponding to $\mathrm{H}$ in the single pulse crater model, $a$ is the height between the highest point of the remaining part of the overlap crater and the bottom of the crater, $r_{c}$ is crater radius, corresponding to $\mathrm{R}$ in the single pulse crater model, $r_{s}$ is the radius of the discharge channel, which is obtained by Eq. 2 .

To make the calculated results more consistent with the actual situation, surface topography parameters of the WEDM continuous discharge material removal model were extracted, as shown in Fig. 11.

The above parameters were processed by means of averaging, and the process was as follows:

The average value of the crater radius is calculated according to Eq. 10.

$\overline{r_{c}}=\frac{\sum_{i=1}^{n} R_{i}}{n}$

The remaining radius of the crater after stacking is calculated according to Eq. 11.

$\bar{s}=\lambda \bar{H}=\lambda \frac{\sum_{i=1}^{n}(H)_{i}}{n}$

The average surface roughness of the model is calculated according to Eq. 12.

$R_{a}=\frac{1}{4}\left[\frac{\overline{r_{c}}+r_{s}}{\overline{r_{c}}}\right]^{2} \cdot \lambda \bar{H}$

Where $\overline{r_{c}}$ is the average radius of the crater $(\mu \mathrm{m})$, $\bar{H}$ is the average depth of the $\operatorname{crater}(\mu \mathrm{m}), \lambda$ is the height residual coefficient, which is related to discharge parameters and obtained by simulation.

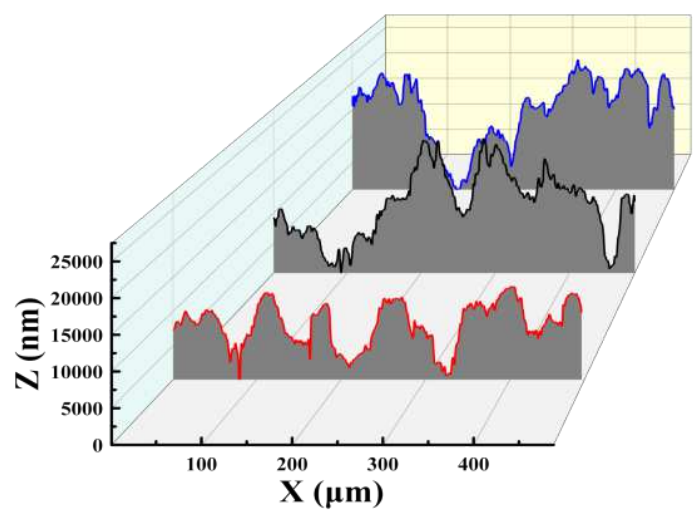

Fig. 11 Section diagram of continuous discharge material removal model extracted randomly.

\section{Experimental details}

The continuous discharge material removal model was verified on the DK7632 high-speed WEDM machine. The dielectric was emulsion and the electrode wire was $0.18 \mathrm{~mm}$ molybdenum wire. The experimental equipment is shown in Fig. 12. The workpiece material is 
EN-GJL-250 (gray cast iron), the dimensions of specimens were $20 \times 20 \times 5 \mathrm{~mm}$. The percentage of chemical composition properties of the workpiece material is given in Table 1. Pulse width $\left(T_{\text {on }} \mu\right.$ s), peak current (Ip A) were used as independent input parameters, and surface roughness $(\mathrm{Ra} \mu \mathrm{m})$ was used as output parameters. The levels of the independent parameters are shown in Table 2. During the experiments, the other parameters, $120 \mathrm{~V}$ open Circuit Voltage, 1:3 Interval Ratio, 4m/min, 5 $\mathrm{kg} / \mathrm{cm} 2$ dielectric flushing pressure, $9 \mathrm{~kg}$ wire tension, $0.18 \mathrm{~mm}$ wire diameter, were kept constant.

As a first step, the surface roughness of each machined material was unified by grinding, and the original surface roughness of each machined material was controlled between $1.3 \mu \mathrm{m}$ and $1.5 \mu \mathrm{m}$. Secondly, in the normal machining process of WEDM, the discharge is continuous and the crater is superimposed on each other, so it is almost impossible to study every single pulse discharge crater. Therefore, to conform to the assumed conditions of the material removal model as much as possible, at the same time, to obtain a single crater. with obvious surface morphology on the continuous electric discharge machining surface, another series of experiments were designed. An often overlooked but important idea is to find the distance between the molybdenum wire and the workpiece at which the spark discharge occurs in the first contact. The motion distance of the molybdenum wire can be detected by using the coordinate motion system of the DK7632 HSWEDM machine. The experimental steps are shown in Fig. 13. Firstly, the initial gap between the molybdenum wire and the workpiece is controlled at $1 \mathrm{~mm}$, and then the molybdenum wire is moved $100 \mu \mathrm{m}$ in turn to make the molybdenum wire gradually close to the workpiece until the discharge spark occurs, and then the discharge machining of the material surface is carried out. Finally, the discharge parameters were adjusted by the WEDM operating panel, and the comparative experiments of nine groups of different discharge parameters were carried out.

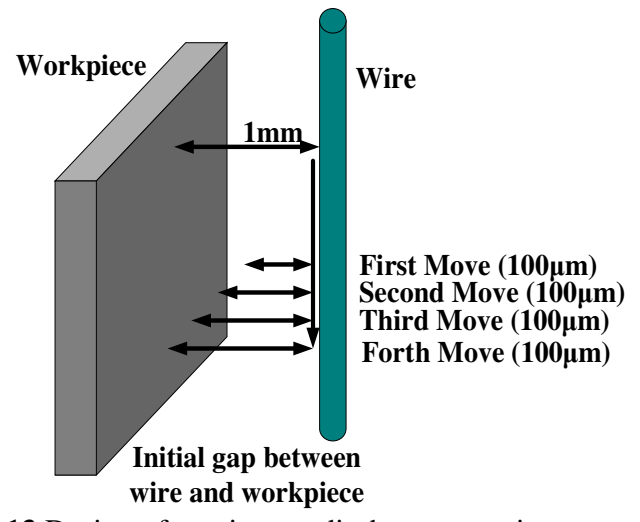

Fig.13 Design of continuous discharge experiment.

White Light Scanning Interferometry. To acquire the average roughness and threedimensional shape image of the material machined surface, we installed the sample on the non-contact sperview W1 optical 3D surface profiler. Images of $489.5 \mu \mathrm{m} \times 489.5 \mu \mathrm{m}$ area were acquired accordingly. Surface Composition Measurement. To measure the composition of the material surface after WEDM, the machined surface was sampled and measured by $\mathrm{x}$-ray diffraction(XRD).

Table 1 Chemical composition properties of EN-GJL-25.

\begin{tabular}{cllllll}
\hline $\begin{array}{c}\text { Chemical } \\
\text { composition }(\%)\end{array}$ & $\mathrm{Fe}$ & $\mathrm{C}$ & $\mathrm{Si}$ & $\mathrm{Mn}$ & $\mathrm{P}$ & $\mathrm{S}$ \\
\cline { 2 - 7 } & 92.50 & 3.50 & 2.50 & 0.75 & 0.45 & 0.20 \\
\end{tabular}


Table 2 Factors and levels for experiment

\begin{tabular}{lllllllllll}
\hline NO. & 1 & 2 & 3 & 4 & 5 & 6 & 7 & 8 & 9 \\
\hline Peak current $(\mathrm{A})$ & 8 & 8 & 8 & 12 & 12 & 12 & 16 & 16 & 16 \\
Pulse width $(\mu \mathrm{s})$ & 12 & 12 & 12 & 24 & 24 & 24 & 32 & 32 & 32 \\
\hline & & & & & & & & & & \\
\hline
\end{tabular}

Fig.12 HS-WEDM experimental equipment diagram.

\section{Experimental results and discussion}

\subsection{D micron scale surface topography based on continuous discharge}

The final surface morphology and crater morphology of each experimental material were analyzed using three-dimensional morphological images. The surface topography and characteristic size parameters of a single crater are shown in Fig. 14. It is found that the radius of the crater is $50-80 \mu \mathrm{m}$, the depth of the crater is $6 \mu \mathrm{m}-10 \mu \mathrm{m}$ and the flanging height is $2 \mu \mathrm{m}$ $8 \mu \mathrm{m}$. This is consistent with the range of characteristic parameters of the single pulse discharge crater model obtained by the previous simulation. Fig. 15 shows the machined surface topography with different discharge parameters.
Obviously, as the discharge energy increases, the volume of material removed from the workpiece increases, as does the size and number of craters on the workpiece surface. At the same time, in the process of crater formation, with the increase of discharge energy, the crater size increase is not synchronized, the crater diameter increase scope is bigger, and the fastest growth rate, then the indentation depth to achieve the stable value, finally because of the effect of processing waste heat of molten material flow trend, flanging height also gradually achieve the stable value. The variation trend of crater size characteristic parameters with discharge energy during simulation is verified.
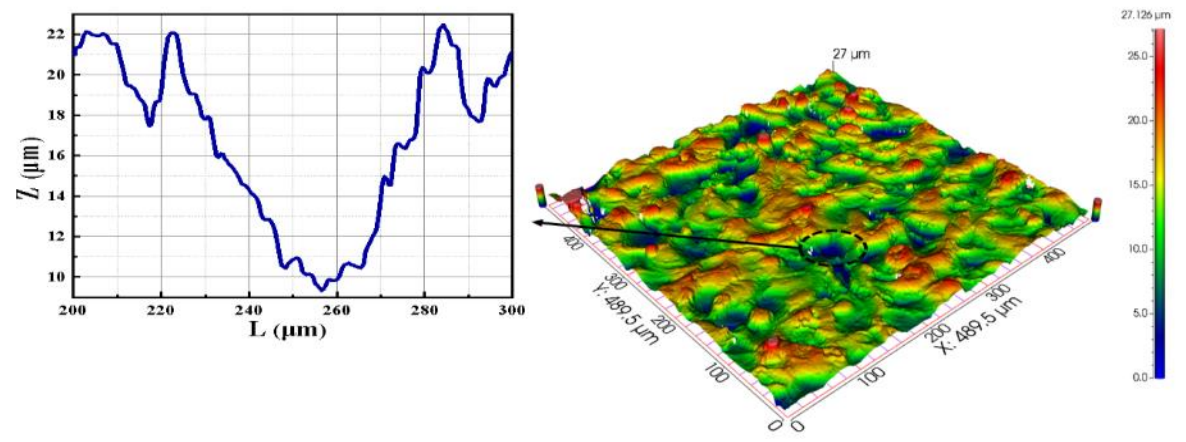
Fig. 14 Surface topography of WEDM continuous discharge: $3 D$ view and crater shape and dimensions $\left(\mathrm{Ip}=8 \mathrm{~A}, \mathrm{~T}_{\mathrm{on}}=12 \mu \mathrm{s}\right)$.
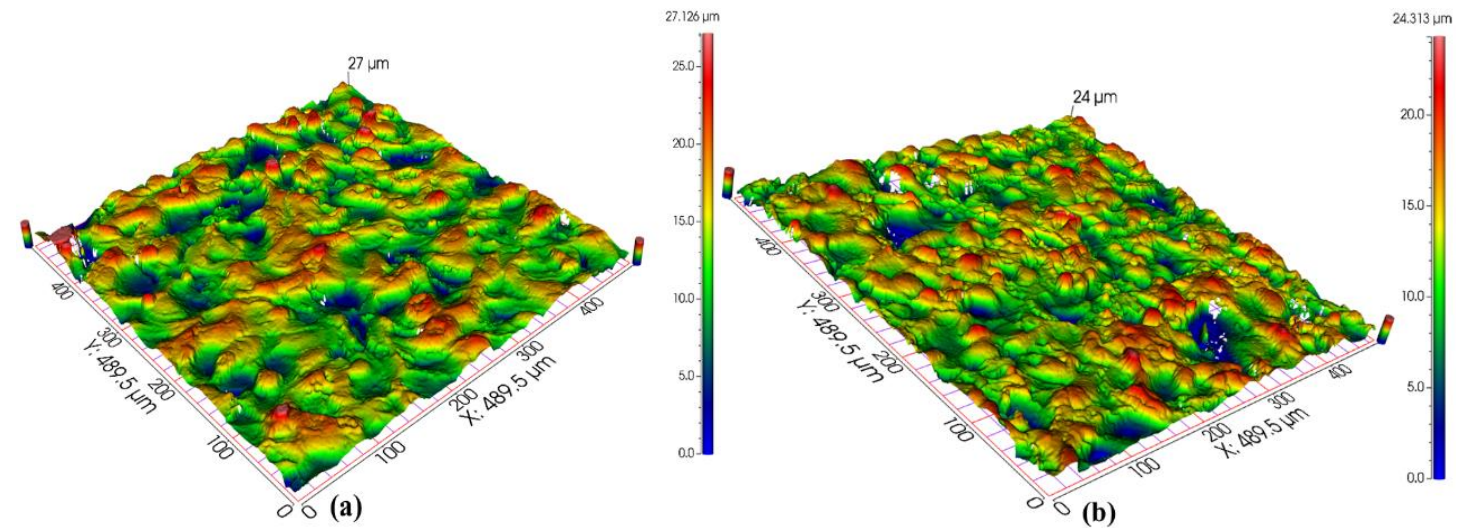

Fig. 15 3D micrometer surface morphology with different discharge energies: (a)Ip=12A, $\mathrm{T}_{\text {on }}=24 \mu \mathrm{s}$; (b)Ip=16A, $\mathrm{T}_{\mathrm{on}}=32 \mu \mathrm{s}$.

After the experiment, the surface of the chemical composition and microstructure of the material was observed by scanning electron microscope (SEM) and XRD measurements surface layer after WEDM machining verified were made. The composition of the surface of the material is shown in Fig. 16. The change of the phase transition of the material surface caused by heat during the simulation process of single pulse discharge.
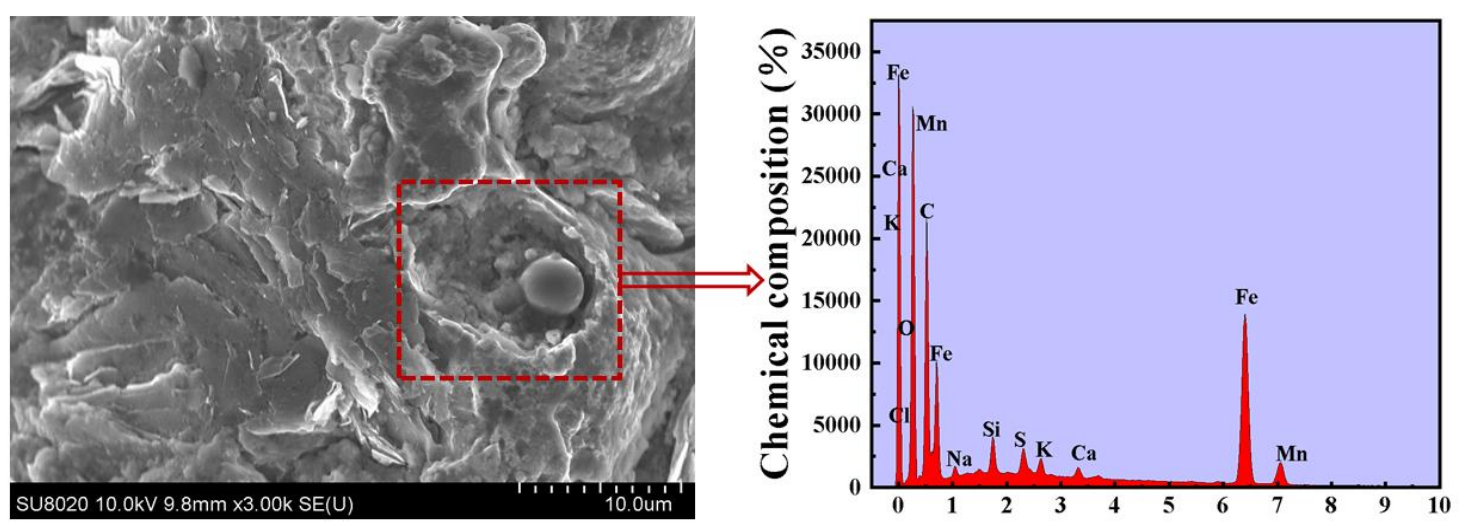

Fig. 16 SEM micrograph and the corresponding XRD spectrum of the micro-crater.

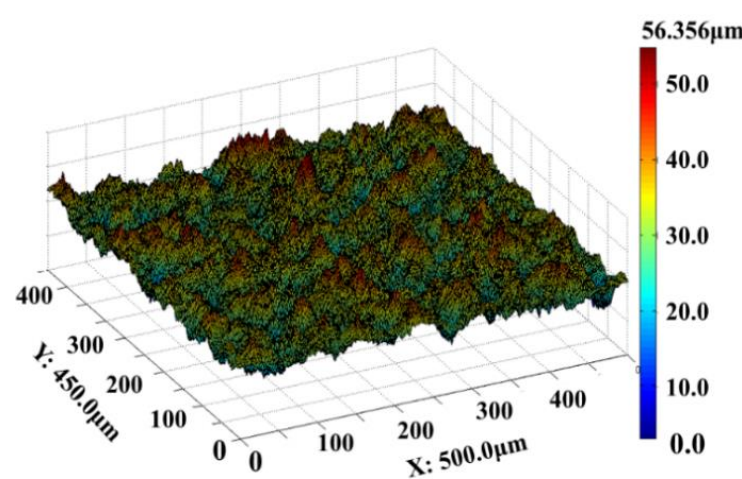

(a)

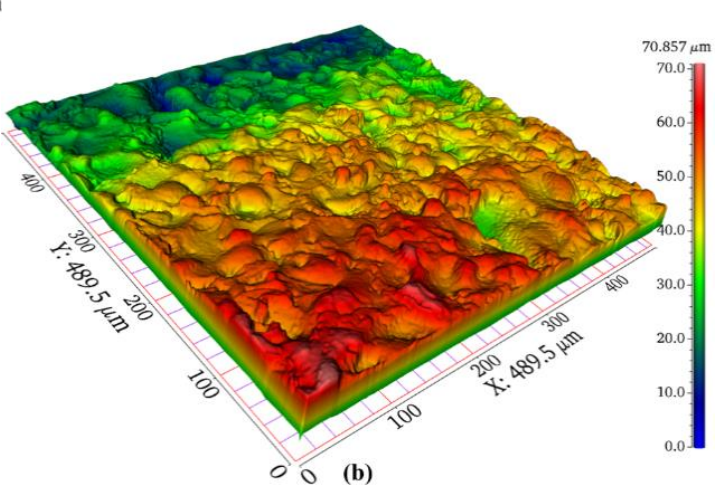

(b)

Fig. 17 Simulated and experimental surface topographies (a) simulated result (b) experimental result. 


\subsection{Model validation}

An example of a simulated surface and an experimental one is shown in Fig. 17. To verify the accuracy of the predicted surface roughness of the material removal model, the actually measured surface roughness is compared with the predicted surface roughness of the model. The surface roughness and average error between the experimental results and the simulation results are shown in Table 3 and Fig. 18. Obviously, the predicted values of the model are basically consistent with the experimental measurements. The average relative error of the WEDM continuous discharge material removal model established in this paper to predict the surface roughness is $8.26 \%$, and there is a certain error between the measured value and the predicted value of the model, because there are many influencing factors in the experiment and modeling process. In the process of single pulse discharge simulation, the grid division, the selection of energy distribution coefficient and convective heat transfer coefficient, and the measurement of thermal properties of EN-GJL250 material at different temperatures have certain influence on the accuracy of simulation results. In the actual machining process of WEDM, the normal discharge, arc discharge and short circuit discharge states are inevitable. However, different types of discharge states have different discharge energy levels. This phenomenon makes the changes of each discharge crater inconsistent, resulting in the increase of surface roughness.
Table 3 Comparison of surface roughness values

\begin{tabular}{cccc}
\hline No. & $\begin{array}{c}\text { Experimental } \\
\mathrm{Ra}(\mu \mathrm{m})\end{array}$ & $\begin{array}{c}\text { Model } \\
\mathrm{Ra}(\mu \mathrm{m})\end{array}$ & Error $(\%)$ \\
\hline 1 & 3.0918 & 2.8974 & 6.29 \\
2 & 3.3579 & 3.3154 & 1.27 \\
3 & 3.5415 & 3.4936 & 1.35 \\
4 & 5.3807 & 4.8681 & 9.53 \\
5 & 6.6678 & 5.4742 & 17.9 \\
6 & 8.0754 & 7.6573 & 5.18 \\
7 & 9.4528 & 8.5294 & 9.77 \\
8 & 10.7634 & 9.9178 & 7.86 \\
9 & 12.0783 & 10.2476 & 15.16 \\
\hline
\end{tabular}

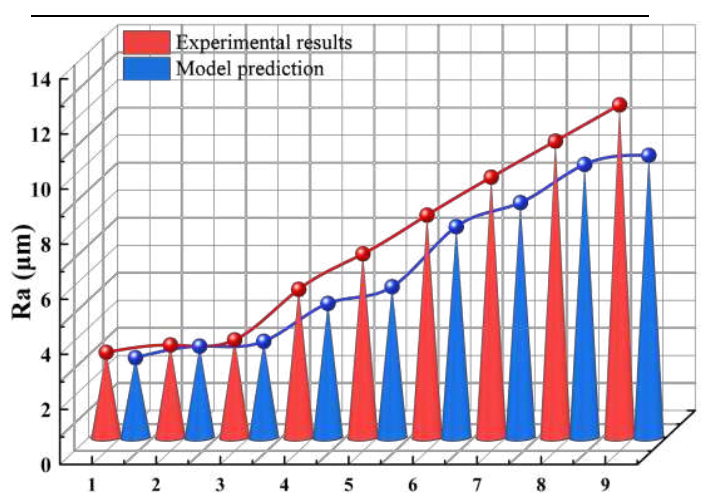

Fig. 18 Comparison of Ra values of simulation and experiment.

\subsection{Influence of discharge parameters on surface roughness}

The relationship between peak current and pulse width and surface roughness can be obtained from Fig. 19. The surface roughness increases with the increase of peak current and pulse width, and the effect of peak current on surface roughness is greater than that of the pulse width. When the discharge current and the increase of pulse width means the increase of single pulse discharging energy, according to the simulation result shows that in front of a single pulse discharge energy increase, in the process of heat transfer of radial direction in the pits in the discharge rate of growth is greater than the depth direction, thus forming the discharge 
crater diameter growth rate is greater than the depth of the growth rate, Finally, the surface roughness increases with the increase of discharge current and pulse width.

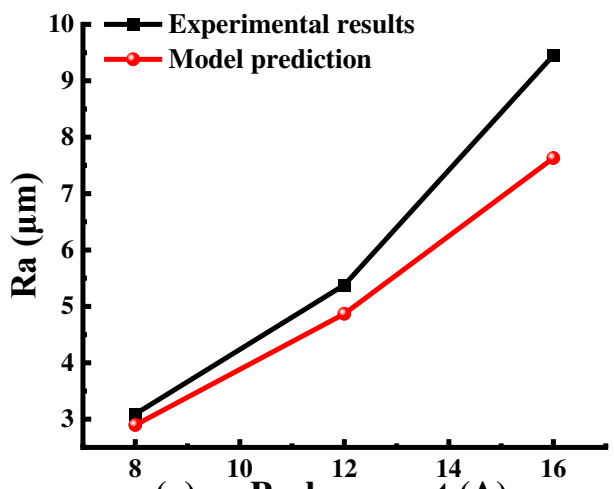

(a) Peak current (A)

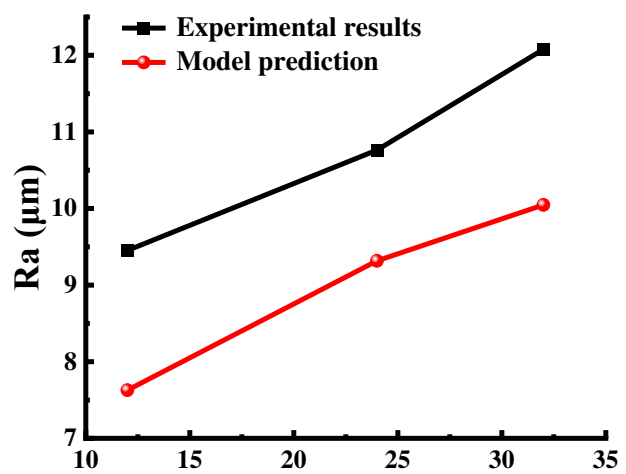

(b) Pulse width ( $\mu$ s)

Fig. 19 (a) the relationship between peak current and surface roughness; (b) the relationship between pulse width and surface roughness.

\section{CONCLUSION}

From the aforementioned analysis and experiments, the following conclusions can be drawn:

(1) In this paper, a thermal-flow coupling model is established in the process of singlepulse discharge. The formation process and morphology evolution process of discharge pits are simulated numerically. The distribution law of temperature field and flow field in the discharge area is studied, and the single-pulse discharge crater model is established. It can be considered that the single pulse crater morphology obtained is more reliable under the influence of considering the molten vaporization or re-solidification of the processed material, the mass loss caused by material evaporation and the residual heat generated by a single spark.

(2) According to the energy conservation theorem, the characteristic size of the impact crater is simulated with different discharge parameters. The effects of peak current and pulse width on temperature and velocity characteristics of discharge pits were analyzed. The results show that the increasing trend of crater radius, depth and flanging height is not synchronous due to the different discharge energy, and the increasing trend of crater radius is more obvious and rapid.

(3) According to the machining mechanism of WEDM, a material removal model with continuous discharge was established to simulate the machining process. The input parameters are peak current and pulse width, and the output parameters are surface roughness. The minimum gap width between the initial surface topography of the workpiece and the electrode wire was taken as the discharge position. Then, the material removal model was tested by experiments. The results show that the prediction accuracy of the WEDM continuous discharge material removal model for surface roughness is $91.74 \%$. The surface roughness increases with the increase of peak current and pulse width, and the effect of the peak current on surface roughness is greater than that of the pulse width.

(4) This study can help to better understand the principle of the WEDM process and the formation of a single pulse discharge crater and accurately predict the final surface morphology of materials after continuous discharge. The machining accuracy and surface integrity of WEDM can be improved by adjusting discharge 
parameters. In order to be closer to the actual machining process, the influence of electric field force and electromagnetic force was added into the model in the subsequent simulation study to simulate the material erosion surface formed in the case of continuous pulse discharge, to achieve more accurate prediction.

\section{LIST OF ABBREVIATIONS}

WEDM = Wire-cut electrical discharge machine

EN-GJL-250 = Gray cast iron material

I $p=$ Peak current

$\mathrm{T}_{\mathrm{on}}=$ Pulse width

$\mathrm{FEM}=$ Finite element method

$\mathrm{Ra}=$ Surface roughness

\section{CONSENT FOR PUBLICATION}

Not applicable.

\section{CONFLICT OF INTEREST}

The authors declare no conflict of interest, financial or otherwise.

\section{FUNDING}

This research was supported by the National Natural Science Foundation of China: 52075134;

Natural Science Foundation of Heilongjiang Province of China: LH2019E062;

Postdoctoral Scientific Research Developmental Fund of Heilongjiang Province of China: LBHQ20097;

Fundamental Research Foundation for Universities of Heilongjiang Province, China: LGYC2018JC040

\section{ACKNOWLEDGEMENTS}

Declared none.

\section{REFERENCES}

[1] Shahri HRF, Mahdavinejad R, Ashjaee M, Abdullah $\mathrm{A}(2017)$ A comparative investigation on temperature distribution in electric discharge machining process through analytical, numerical and experimental methods. Int. J. Mach. Tools Manuf 114:35-53.
[2] Bilal A, Jahan M, Talamona D, Perveen A(2019) Electro-discharge machining of ceramics: A review. Micromachines 10:10.

[3] Sabyrov N, Jahan M, Bilal A, Perveen A(2019) Ultrasonic vibration assisted electro-discharge machining (edm) - An overview. Materials 12:522.

[4] Das SS, Patowari PK (2018) Fabrication of serpentine micro-channels on glass by ultrasonic machining using developed micro-tool by wire-cut electric discharge machining. Int $\mathrm{J}$ Adv Manuf Technol 95(5-8):3013-3028.

https://doi.org/10.1007/s00170-017-1441-4

[5] Qudeiri JEA, Mourad AHI, Ziout A, Abidi MH, Elkaseer A (2018) Electric discharge machining of titanium and its alloys. The international journal of advanced manufacturing technology 96(1): 1319-1339. [6] Tsai TC, Horng JT, Liu NM, Chou CC, Chiang, KT (2008) The effect of heterogeneous second phase on the machinability evaluation of spheroidal graphite cast irons in the WEDM process. Materials \& Design 29(9):1762-1767.

https://doi.org/10.1016/j.matdes.2008.03.026.

[7] El-Mahalawy M, Samuel M, Fouda N, El-Bahloul S (2021) Investigating the Effect of Wire Electrical Discharge Machining Factors for Ductile Cast Iron (ASTM A536). International Journal of Industrial Engineering \& Production Research 33(2): 0-0.

[8] Gangil M, Pradhan MK (2017) Modeling and optimization of electrical discharge machining process using RSM: a review. Materials Today: Proceedings 4(2): 1752-1761.

[9] Gowthaman PS, Jeyakumar S (2019) A Review on machining of High Temperature Aeronautics Superalloys using WEDM. Materials Today: Proceedings 18:4782-4791.

[10] Mohanty CP, Mahapatra SS, Sahu J (2016) Parametric optimization of electrical discharge machining process: a numerical approach. Int J Ind Syst Eng 22: 207.

[11] Kumar S, Grover S, Walia RS (2018) Effect of hybrid wire EDM conditions on generation of residual stresses in machining of $\mathrm{HCHCr}$ D2 tool steel under ultrasonic vibration. Int J Interact Des Manuf 12(3): 
1119-1137.

https://doi.org/10.1007/s12008-018-0474-8

[12] Sanchez JA, Conde A, Arriandiaga A, Wang J, Plaza S (2018) Unexpected event prediction in wire electrical discharge machining using deep learning techniques. $\quad$ Materials $11(7)$ : 1100. https://doi.org/10.3390/ma11071100

[13] Sharma P, Chakradhar D, Narendranath S (2018) Analysis and optimization of WEDM performance characteristics of Inconel 706 for aerospace application. Silicon 10(3): 921-930. https://doi.org/10.1007/s12633-017-9549-6.

[14] Vignesh M, Ramanujam R (2018) Response optimization in wire electrical discharge machining of AISI H11 tool steel using Taguchi - GRA approach. Int J Mach Mater 20: 474-495.

[15] Kunieda M, Lauwers B, Rajurkar KP, Schumacher BM (2005) Advancing EDM through fundamental insight into the process. CIRP Ann 54: 64-87.

[16] Chiang KT, Chang FP (2006) Optimization of the WEDM process of particle-reinforced material with multiple performance characteristics using grey relational analysis. Journal of Materials Processing Technology 180(1-3): 96-101.

[17] Chaudhari R, Vora JJ, Mani Prabu SS, Palani IA, Patel VK, Parikh DM, Lacalle LNL (2019) Multiresponse optimization of WEDM process parameters for machining of super-elastic nitinol shape-memory alloy using a heat-transfer search algorithm. Materials 12(8): 1277.

[18] Tao J, Ni J, Shih AJ (2012) Modeling of the anode crater formation in electrical discharge machining. Journal of Manufacturing Science and Engineering-Transactions of the Asme 134(1): 11

[19] Yue XM, Yang XD (2017) Molecular dynamics simulation of material removal process and crystal structure evolution in EDM with discharge on different crystal planes. Int J Adv Manuf Technol 92(9-12): 3155-3165

[20] Vignesh M, Ramanujam R (2021) Numerical modelling and experimental validation of crater formation in WEDM hybrid turning of Ti-6Al-4V alloy. Proceedings of the Institution of Mechanical Engineers, Part E: Journal of Process Mechanical Engineering 235(2): 392-404.

[21] Esteves PM, Wiessner M, Costa JV, Sikora M, Wegener K (2021) WEDM single crater asymmetry. The International Journal of Advanced Manufacturing Technology, p1-7.

[22] Maher I, Sarhan AA (2017) Proposing a new performance index to identify the effect of spark energy and pulse frequency simultaneously to achieve high machining performance in WEDM. The International Journal of Advanced Manufacturing Technology 91(1): 433-443.

[23] Chen Z, Zhang GJ, Han FL, Zhang YM, Rong YM (2018) Determination of the optimal servo feed speed by thermal model during multi-pulse discharge process of WEDM. Int J Mech Sci 142: 359-369

[24] Nain SS, Garg D, Kumar S (2017) Prediction of the performance characteristics of WEDM on UdimetL605 using different modelling techniques. Materials Today: Proceedings 4(2): 546-556.

https://doi.org/10.1016/j.matpr.2017.01.056.

[25] Kumar H, Manna A, Kumar R (2018) Modeling of process parameters for surface roughness and analysis of machined surface in WEDM of $\mathrm{Al} / \mathrm{SiC}$ MMC. Transactions of the Indian Institute of Metals 71(1): 231-244.

[26] Izquierdo B, Sanchez JA, Plaza S, Pombo I, Ortega N (2009) A numerical model of the EDM process considering the effect of multiple discharges. International Journal of Machine Tools and Manufacture 49(3-4): 220-229.

[27] Jithin S, Raut A, Bhandarkar UV, Joshi SS (2018) Fe modeling for single spark in edm considering plasma flushing efficiency. Procedia Manufacturing 26: 617-628.

[28] Wang Y, Chen SY, Xiong W, Wu CZ (2020) Study on workpiece surface forming mechanism by successive discharges during MSV-MF complexassisted WEDM-LS process. The International Journal of Advanced Manufacturing Technology 108(9):29853000 .

[29] Vishwa B, Rao P S. Gaussian Energy Distribution 
of a RC-Circuit Based Single Discharge Pulse in Micro-EDM. International Journal of Innovative Technology and Exploring Engineering 8(10): 43474350.

[30] Parasrampuria AS, (2020) Single Spark Numerical Modelling of Wire Electrical Discharge Machining for Solar Photovoltaic Applications.

[31] Ikai T, Fujita I, Hashiguchi K (1992) Heat input radius for crater formation in the electric discharge machining. IEEJ Trans. Ind. Appl 112:943-949.

[32] Shabgard MR, Gholipoor A, Mohammadpourfard M (2018) Numerical and experimental study of the effects of ultrasonic vibrations of tool on machining characteristics of EDM process. International Journal of Advanced Manufacturing Technology 96(5-8): 2657-2669.

[33] Razeghiyadaki A, Molardi C, Talamona D, Perveen A (2019) Modeling of material removal rate and surface roughness generated during electrodischarge machining. Machines 7(2): 47.

[34] Ulas M, Aydur O, Gurgenc T, Ozel C (2020) Surface roughness prediction of machined aluminum alloy with wire electrical discharge machining by different machine learning algorithms. Journal of Materials Research and Technology 9(6): 1251212524.

https://doi.org/10.1016/j.jmrt.2020.08.098

[35] Razeghiyadaki A, Molardi C, Talamona D, Perveen A (2019) Modeling of Material Removal Rate and Surface Roughness Generated during ElectroDischarge Machining. Machines 7(2): 47. https://doi.org/10.3390/machines7020047

[36] Ikai T, Fujita I, Hashiguchi K (1992) Heat input radius for crater formation in the electric discharge machining. IEEJ Transactions on Industry Applications 112(10): 943-949.

[37] Salonitis K, Stournaras A, Stavropoulos P, Chryssolouris G (2009) Thermal modeling of the material removal rate and surface roughness for die- sinking EDM. The International Journal of Advanced Manufacturing Technology, 40(3): 316-323.

[38] Zhang QH, Du R, Zhang JH, Zhang QB (2006) An investigation of ultrasonic-assisted electrical discharge machining in gas. International Journal of Machine Tools and Manufacture, 46(12-13): 15821588. 